\title{
Cranial Nerve Palsy after Embolization of Giant Cavernous Carotid Aneurysm with Onyx HD-500: Case Series and Review of the Literature
}

\author{
Sonia Teufack, MD, Stavropoula Tjoumakaris, MD, Fernando Gonzalez, MD, \\ Aaron Dumont, MD, Robert Rosenwasser, MD, Pascal Jabbour, MD \\ Department of Neurological Surgery, Thomas Jefferson University, Philadelphia, PA
}

Running title: Onyx HD-500 embolization of giant cavernous aneurysms.

\author{
Abstract \\ Background \\ Onyx liquid embolic system has been used in the treatment of intracranial aneurysm and arterio- \\ venous malformation since 1999. Recently, Onyx HD-500 has been especially advocated for the \\ treatment of large and giant intracranial aneurysms.
}

\section{Methods}

Two cases of cranial nerve palsy following embolization of unruptured symptomatic giant cavernous internal carotid artery aneurysms are presented. The first patient had a right $2.0 \mathrm{~cm} \times 1.5 \mathrm{~cm}$ cavernous aneurysm, and the second a $2.7 \mathrm{~cm} \times 1.8 \mathrm{~cm}$ left cavernous aneurysm. Both patients developed persistent diplopia after embolization with Onyx HD-500.

\section{Results}

Ophthalmological evaluation revealed that both patients had abductor nerve palsy on the same side as the aneurysm; in addition the second patient also had ipsilateral occulomotor nerve palsy.

\section{Conclusion}

Embolization of giant cavernous aneurysm with Onyx HD-500 can result in worsening or development of new cranial nerve palsies; possibly from mass effect resulting from edema and thrombosis of the aneurysm in the cavernous sinus.

\section{Keywords}

Onyx HD-500, giant aneurysm, cranial nerve palsy.

\section{Introduction}

Onyx HD-500 is a biocompatible non-adhesive liquid embolic agent composed of $20 \%$ ethylene- vinyl alcohol (EVOH) copolymer and dimethyl sulfoxide (DMSO) solvent added to micronized tantalum powder, a high- molecular- weight metal that renders the solution radiopaque ${ }^{3}$. Onyx HD-500 differs from other liquid embolic systems in that it has the highest polymer concentration at $20 \%$, versus $12 \%$ in the original formulation for aneurysm treatment. Higher polymer concentration results in higher viscosity thus lowering the chance of reflux in parent vessels.

\section{Case Report}

Case 1

A 60-year-old female initially presented to her primary care physician with complains of fatigue and left hemibody numbness. Her evaluation included magnetic resonance imaging of the brain, which led to the incidental diagnosis of a $1.5 \mathrm{~cm}$ right cavernous ICA aneurysm. The patient was subsequently lost to follow-up and presented again 5 years later when she started noticing episodes of blurry vision. At this time, neurological and ophthalmological evaluation did not reveal any permanent deficit. All cranial nerves were intact, visual fields were full, and visual acuity was 20/30 in both eyes. A repeat $\mathrm{MR}$ angiogram revealed that the aneurysm increased in size to $1.8 \mathrm{~cm}$. After a discussion of the risks and benefit of surgery, the patient opted for endovascular intervention. She underwent balloon-assisted glue embolization of the aneurysm. Complete obliteration of the aneurysm was achieved with $4.8 \mathrm{~mL}$ of Onyx HD-500. There were no intra-operative complications. However, the patient developed diplopia secondary to partial abducens nerve palsy postoperatively. The patient was treated with a fast steroid taper. At her 3 months follow-up, she was noted to have persistence of the partial right abducens nerve palsy.

\section{Case 2}

A 58-year-old male with progressive memory difficulty was evaluated with MR imaging of the brain by his primary care physician. This workup led to the incidental diagnosis of a $1.3 \mathrm{~cm}$ left cavernous-ICA aneurysm. At the time, patient denied any headache or vision changes. After discussion of management options, the patient and family decided to have the aneurysm followedup. Over the next three years, the aneurysm remained stable on serial MRA. However, on subsequent follow-up, patient reported an acute persistent diplopia which was more pronounced with left gaze. On examination, the patient was found to have partial left abducens nerve palsy; his neurological and ophthalmological exams were otherwise normal. This instance, an MRA revealed a significant increase in the size of the left cavernous-ICA aneurysm to $2.7 \mathrm{~cm}$. After discussion of the risks and benefit of surgery, the patient underwent balloon-assisted glue embolization of the aneurysm. Complete obliteration was achieved with $8.4 \mathrm{~mL}$ of Onyx HD-500 (see Figure 1); there were no intra-operative complications. Post-operatively, the patient developed worsening of his diplopia. Neurological exam revealed complete left abducens nerve palsy and partial left occulomotor nerve palsy. After a short course of steroid, the patient had resolution of the left occulomotor nerve palsy. However, he had persistence of the left abducens palsy through his last visit with us for a six-week follow-up. 


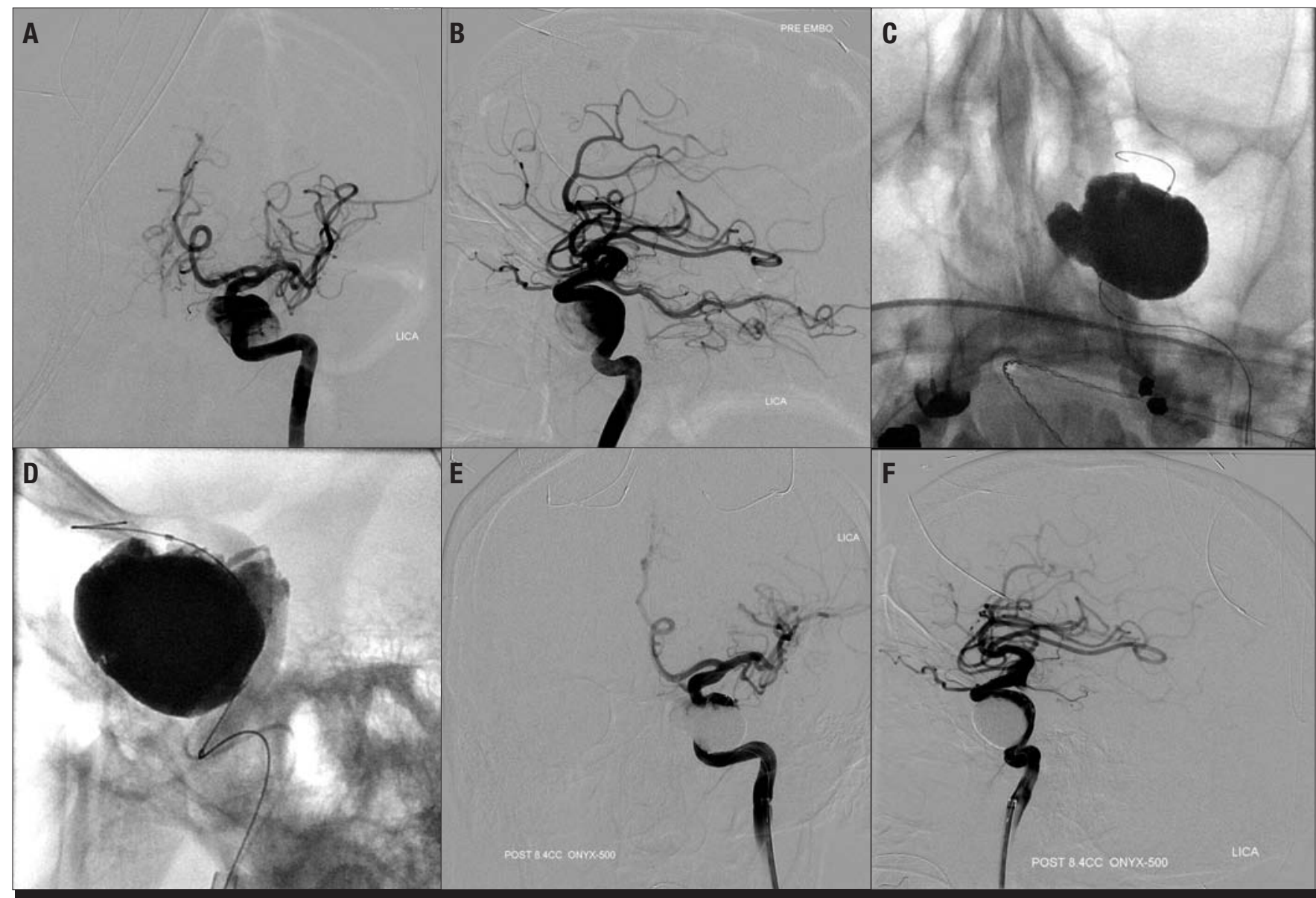

Figure 1

(A, B) AP/Lateral views of a $2.7 \mathrm{~cm}$ left cavernous carotid aneurysm; (C, D) AP/Lateral views Onyx HD-500 injection; (E, F) AP/Lateral views post embolization with $100 \%$ occlusion.

\section{Discussion}

The primary goal of intracranial un-ruptured aneurysm treatment is complete occlusion to avoid the potentially high morbidity and mortality associated with acute rupture. Several studies have shown that incomplete occlusion following open surgical and endovascular treatment of aneurysms is associated with rebleeding. ${ }^{6,713}$ Since their introduction in the 1990s, GDC coils have grown in popularity are now accepted as a reasonable endovascular treatment modality for intracranial aneurysms. Nevertheless, initial studies showed that endovascular coiling with regular platinum coils had a higher rate of incomplete occlusion and recanalization when used for wide neck aneurysms (with a neck larger than $4 \mathrm{~mm}$ and a dome to neck ratio less than 1.5) as well as for large and giant aneurysms. ${ }^{4,5,8}$ Since then, newer technologies have been developed to improve thrombosis of the dome and neck of the aneurysm, while preserving the parent vessels integrity. These technologies include stent-assisted coiling, balloon assisted coiling and embolization with a liquid embolic agent such as Onyx HD-500.

Onyx HD-500 is thought to be particularly applicable to the treatment of wide neck and large aneurysms; it has been shown to have a higher rate of complete occlusion..$^{10,12}$ Since it is a liquid material, it can be made to fill close to $100 \%$ of the aneurysm capacity; the use of balloon assistance technique allows for modeling of the cast to preserve the parent vessel. In addition, because of its high viscosity, Onyx HD-500 has a lower chance of refluxing into the parent vessel and causing thrombo-embolic events. Another important characteristic of Onyx HD-500 is its ability to induce a stronger neo-epithelialization reaction at the neck of the aneurysm. .10

Few studies have reported complications from mass effect of the liquid embolic agents on adjacent structures. They mainly described cranial nerve palsies, ${ }^{2,10,12}$ and in rare instances, motor deficits from compression of critical intracranial structures. ${ }^{11}$ Cranial nerves commonly involved are the optic nerve with supraclinoidalICA aneurysms; the occulomotor and abducens nerves for cavernous-ICA aneurysms. In their series, Weber et $\mathrm{al}^{12}$ presented six cases of cavernous-ICA aneurysms treated with Onyx HD-500. One patient had persistence of his cranial nerve palsy; two patients had improvement overtime, and two had complete resolution. One patient was asymptomatic. Piske et $\mathrm{al}^{10}$ series included six cavernous-ICA aneurysms 
as well. Three patients had improvement in their cranial nerve palsy at six month, versus three with no improvement. The results of our series corroborate previous reports. One of our patients had no change in her cranial palsy, and the other had some improvement in the subacute phase, with chances of further recovery. We would argue that the combination of high volume in addition to high density of liquid embolic material required for the complete obliteration of large to giant aneurysms might result in significant mass effect. Such is the case particularly for cavernous-ICA aneurysm. As previously speculated by some authors, ${ }^{1,10}$ we put forward that the use of a combination of special coil plus liquid embolic material might have better outcome for the treatment of such aneurysms.

\section{Conclusion}

Onyx HD-500 is a liquid embolic agent with high viscosity reducing the chance of reflux of embolic material into the parent vessel. This property makes it a reasonable option for embolization of larger and wider neck aneurysms. However, in the specific instance of large and giant cavernous-ICA aneurysms, the combination of high volume and high density might result in mass effect and cranial nerve deficits. For such aneurysms, consideration might be given for combined coiling and liquid embolization.

\section{References}

1. Cekirge HS, Saatci I, Geyik S, Yavuz K, Ozturk H, Pamuk G: Intrasaccular combination of metallic coils and onyx liquid embolic agent for the endovascular treatment of cerebral aneurysms. J Neurosurg 105:706-712, 2006.

2. Cekirge HS, Saatci I, Ozturk MH, Cil B, Arat A, Mawad M, Ergungor F, Belen D, Er U, Turk S, Bavbek M, Sekerc Z, Beskonakli E, Ozcan OE, Ozgen T: Late angiographic and clinical follow-up results of 100 consecutive aneurysms treated with Onyx reconstruction: largest single-center experience. Neuroradiology 48:113-126, 2006.

3. EV3 The Endovascular Company: Onyx HD-500, 2007

4. Grunwald IQ, Papanagiotou P, Struffert T, Politi M, Krick C Gul G, Reith W: Recanalization after endovascular treatmen of intracerebral aneurysms. Neuroradiology 49:41-47, 2007.

5. Henkes H, Fischer S, Weber W, Miloslavski E, Felber S, Brew S, Kuehne D: Endovascular coil occlusion of 1811 intracranial aneurysms: early angiographic and clinical results. Neurosurgery 54:268-80; discussion 280-5, 2004.

6. Molyneux A, Kerr R, Stratton I, Sandercock P, Clarke M, Shrimpton J, Holman R, International Subarachnoid Aneurysm Trial (ISAT) Collaborative Group: International Subarachnoid Aneurysm Trial (ISAT) of neurosurgical clipping versus endovascular coiling in 2143 patients with ruptured intracranial aneurysms: a randomised trial. Lancet $360: 1267-1274,2002$

7. Molyneux AJ, Cekirge S, Saatci I, Gal G: Cerebral Aneurysm Multicenter European Onyx (CAMEO) trial: results of a prospective observational study in 20 European centers. AJNR Am J Neuroradiol 25:39-51, 2004.

8. Moret J, Cognard C, Weill A, Castaings L, Rey A: Reconstruction technic in the treatment of wide-neck intracranial aneurysms. Long-term angiographic and clinical results. Apropos of 56 cases. J Neuroradiol 24:30-44, 1997.

9. Murayama Y, Vinuela F, Tateshima S, Vinuela F,Jr, Akiba Y: Endovascular treatment of experimental aneurysms by use of a combination of liquid embolic agents and protective devices. AJNR Am J Neuroradiol 21:1726-1735, 2000.
10. Piske RL, Kanashiro LH, Paschoal E, Agner C, Lima SS, Aguiar PH: Evaluation of Onyx HD-500 embolic system in the treatment of 84 wide-neck intracranial aneurysms. Neurosurgery 64:E865-75; discussion E875, 2009.

11. Van Loock K, Menovsky T, Voormolen MH, Plazier M, Parizel P, De Ridder D, Maas AI, Hernesniemi JA: Microsurgical removal of Onyx HD-500 from an aneurysm for relief of brainstem compression. J Neurosurg, 2009.

12. Weber W, Siekmann R, Kis B, Kuehne D: Treatment and follow-up of 22 unruptured wide-necked intracranial aneurysms of the internal carotid artery with Onyx HD 500. AJNR Am J Neuroradiol 26:1909-1915, 2005.

13. Wermer MJ, Greebe P, Algra A, Rinkel GJ: Incidence of recurrent subarachnoid hemorrhage after clipping for ruptured intracranial aneurysms. Stroke 36:2394-2399, 2005.

\section{Disclosures/Disclaimers}

We do not have any disclosure to present and no conflict of interest.

\section{Corresponding Author}

Sonia Teufack, MD

Department of Neurological Surgery

Thomas Jefferson University

909 Walnut St, 2nd Floor

Philadelphia, PA 19107

Email: sonia.teufack@jeffersonhospital.org

Phone: 215-687-8782

Fax: 215-503-2452

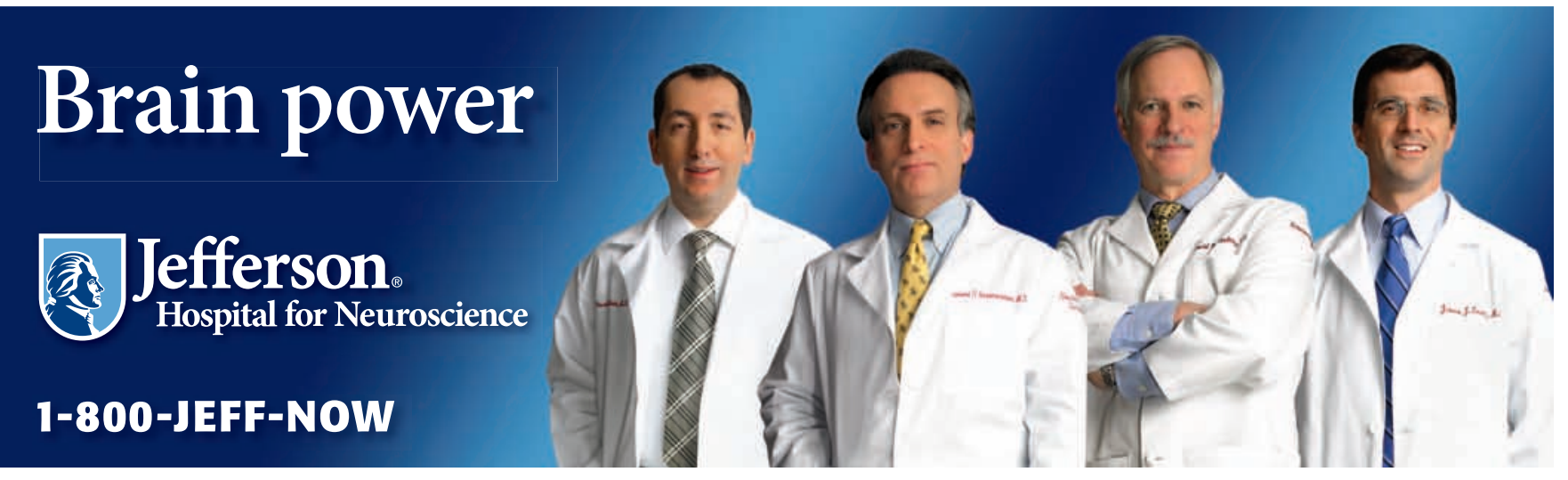

